\title{
A SHORT PROOF OF BERGER'S CURVATURE TENSOR ESTIMATES
}

\section{HERMANN KARCHER}

ABSTRACT. A simple proof of Berger's estimate of the curvature tensor components in terms of the pinching is given and the result is extended to nonorthonormal arguments of the curvature tensor.

Let $M$ be a Riemannian manifold with scalar product $(;)$ and curvature tensor $R: T M \times T M \rightarrow T M$. The sectional curvatures of $M$ at $p$ are given by

(1) $K(u, v)=(R(u, v) v ; u) \cdot\left((u ; u)(v ; v)-(u ; v)^{2}\right)^{-1}$, for independent $u, v \in M_{p}$.

Put

(2) $\delta=\min _{u, v \in M_{p}} K(u, v), \Delta=\max _{u, v \in M_{p}} K(u, v), \bar{K}=\max (|\delta|,|\Delta|)$. $(\Delta-\delta) \cdot \bar{K}^{-1}$ is the pinching of $M$.

Berger's result [1], [2] (orginally for $\delta \geqq 0$, but this is not needed as Tsagas [4] observed) is

(3) $|(R(u, v) v ; w)| \leqq \frac{1}{2}(\Delta-\delta)$ for orthonormal $u, v, w \in M_{p}$,

(4) $|(R(u, v) x ; w)| \leqq \frac{2}{3}(\Delta-\delta)$ for orthonormal $u, v, w, x \in M_{p}$.

The constants $\frac{1}{2}$ and $\frac{2}{3}$ cannot be improved. Apply Theorem 3.2.7 of $\left[2\right.$, p. 92] to $C P^{n}$.

ProOF OF (3) AND (4). We use the curvature tensor identities

(5) $4(R(u, v) v ; w)=(R(u+w, v) v ; u+w)-(R(u-w, v) v ; u-w)$,

$$
\begin{aligned}
6(R(u, v) x ; w)= & (R(u, v+x) v+x ; w)-(R(u, v-x) v-x ; w) \\
& -(R(v, u+x) u+x ; w)+(R(v, u-x) u-x ; w),
\end{aligned}
$$

which are immediate from the linearity and symmetries of $R$. (5) and (6) express $(R(u, v) x ; w)$ in a simpler way in terms of sectional curvatures than is given in $[3$, p. 93].

For or thonormal $u, v, w$ we have $(u \pm w)^{2}=2$, hence (3) is clear from (5), (1), (2). If $u, v, w, x$ are orthonormal then (3) applies to the four terms on the right of (6); together with $(v \pm x)^{2}=(u \pm x)^{2}=2$ we have (4).

To formulate the extensions of (3) and (4) we interpret the endpoints of three unit vectors $u, v, w \in M_{p}$ as vertices of a spherical tri-

Received by the editors February 26, 1970.

AMS 1969 subject classifications. Primary 5370, 5342.

Key words and phrases. Curvature tensor identities, sectional curvature, pinching, curvature tensor norm, estimation of curvatures tensor components. 
angle. With the notation $(u ; v)^{2}=\cos ^{2}(u, v)=1-\sin ^{2}(u, v)$ the spherical cosine formula reads

$$
\cos (u, w)=\cos (u, v) \cdot \cos (v, w)+\sin (u, v) \cdot \sin (v, w) \cdot \cos \angle u v w .
$$

THEOREM. The following generalization of (3) and (4) holds (for unit vectors $u, v, w, x \in M_{p}$ )

$$
\begin{aligned}
\mid R(u, v) v ; w) \mid \leqq & |\sin (u, v) \cdot \sin (v, w)| \\
& \cdot\left\{\frac{1}{2}(\Delta-\delta)|\sin \angle u v w|+\bar{K} \cdot|\cos \angle u v w|\right\} \\
\leqq & \left(\bar{K}^{2}+\frac{1}{4}(\Delta-\delta)^{2}\right)^{1 / 2},
\end{aligned}
$$

where $\cos \angle u v w=0$ if $w \perp u, v$.

(8) $|(R(u, v) x ; w)| \leqq|\sin (u, v) \cdot \sin (x, w)| \cdot \frac{2}{3}(\Delta-\delta)$, for $w \perp u, v$.

$$
|(R(u, v) x ; w)| \leqq\left(\bar{K}^{2}+(25 / 36)(\Delta-\delta)^{2}\right)^{1 / 2},
$$

hence, if $\|R\|$ denotes the norm of $R$ as quadrilinear map then $\|R\|$ $\leqq\left(\bar{K}^{2}+(25 / 36)(\Delta-\delta)^{2}\right)^{1 / 2}$.

Proof. Put $y=u-v(u ; v), z=w-v(w ; v)$, then $|y|=|\sin (u, v)|$, $|z|=|\sin (w, v)|$ and $v \perp y, z$. Therefore we can apply (3) to the first term on the right of

$$
\begin{aligned}
(R(u, v) v ; w)= & \left(R(y, v) v ; z-\frac{y}{|y|^{2}}(y ; z)\right) \\
& +\left(R(y, v) v ; \frac{y}{|y|^{2}}(y ; z)\right)
\end{aligned}
$$

and get the first part of (7) since-by the spherical cosine formula-

and

$$
|\cos (y, z)|=\frac{|(y ; z)|}{|y||z|}=|\cos \angle u v w|
$$

$$
\begin{aligned}
|y| \cdot\left|z-\frac{y}{|y|^{2}}(y ; z)\right| & =|y||z||\sin (y, z)| \\
& =|\sin (u, v) \cdot \sin (v, w) \cdot \sin \angle u v w| .
\end{aligned}
$$

The second part of (7) follows from $|A \cdot \sin \alpha|+|B \cdot \cos \alpha|$ $\leqq\left(A^{2}+B^{2}\right)^{1 / 2}$. The sin-factors in (8) are trivial since with

$u^{\prime}=(u-v(u ; v)) \cdot|\sin (u, v)|^{-1}, \quad x^{\prime}=(x-w(x ; w)) \cdot|\sin (x, w)|^{-1}$

we have

$$
(R(u, v) x ; w)=|\sin (u, v) \cdot \sin (x, w)| \cdot\left(R\left(u^{\prime}, v\right) x^{\prime} ; w\right) .
$$


We apply (7) to the four terms on the right of (6) (with $u^{\prime}, x^{\prime}$ instead of $u, x)$ and since $w \perp u^{\prime}, v, x^{\prime}$ all the $\bar{K} \cdot \cos$-terms vanish. Hence (8) follows using $\left(v+x^{\prime}\right)^{2}+\left(v-x^{\prime}\right)^{2}+\left(u^{\prime}+x^{\prime}\right)^{2}+\left(u^{\prime}-x^{\prime}\right)^{2}=8$. In (9) we can assume $u \perp v, x \perp w$ (replace $u, x$ by $\left.u^{\prime}, x^{\prime}\right)$. Put $y=u-x(u ; x)$, $z=v-x(v ; x), a=u(v ; x)-v(u ; x)$. Note $a \perp x$ and $y(v ; x)-v(u ; x)$ $=a-x(u ; x)(v ; x)$. Then

$$
\begin{aligned}
(R(u, v) x ; w) & =(R(y, z) x ; w)+(R(y, x(v ; x)) x ; w)+(R(x(u ; x), v) x ; w) \\
& =(R(y, z) x ; w)+(R(a, x) x ; w) .
\end{aligned}
$$

From (8) we have

$$
\begin{aligned}
|(R(y, z) x ; w)| & \leqq \frac{2}{3}(\Delta-\delta)|y||z||\sin (y, z)| \\
& =\frac{2}{3}(\Delta-\delta)|\sin (u, x) \cdot \sin (v, x) \cdot \sin \angle u x v|,
\end{aligned}
$$

and from (7) we have

$$
|(R(a, x) x ; w)| \leqq|a| \cdot\left(\bar{K}^{2}+\frac{1}{4}(\Delta-\delta)^{2}\right)^{1 / 2} .
$$

Observe $|a|^{2}=(u ; x)^{2}+(v ; x)^{2} \leqq|x|^{2}=1$ and-from the spherical cosine formula for the triangle $u, x, v-$

$$
|\sin (u, x) \cdot \sin (v, x) \cdot \sin \angle u x v|^{2}=1-|a|^{2} .
$$

Then (9) follows again from $|A \cdot \sin \alpha|+|B \cdot \cos \alpha| \leqq\left(A^{2}+B^{2}\right)^{1 / 2}$.

\section{REFERENCES}

1. M. Berger, Sur quelques variêtés riemanniennes suffisamment pincées, Bull. Soc. Math. France 88 (1960), 57-71. MR 24 \#A3606.

2. S. I. Goldberg, Curvature and homology, Pure and Appl. Math., vol. XI, Academic Press, New York, 1962. MR 25 \#2537.

3. D. Gromoll, W. Klingenberg and W. Meyer, Riemannsche Geometrie im Grossen, Lecture Notes in Math., no. 55, Springer-Verlag, Berlin and New York, 1968. MR 37 \#451.

4. G. Tsagas, $A$ relation between Killing tensor fields and negative pinched Riemannian manifolds, Proc. Amer. Math. Soc. 22 (1969), 476-478. MR 39 \#3429.

Mathematisches Institut, Universität Bonn, Bonn, Germany 